\title{
FATORES CRÍTICOS NO GERENCIAMENTO DE PROJETOS PÚBLICOS SUSTENTÁVEIS
}

\section{CRITICAL FACTORS IN THE MANAGEMENT OF SUSTAINABLE PUBLIC PROJECTS}

\author{
Damila Paulus Link \\ Mestre em Ciências Contábeis e Administração \\ Universidade Comunitária da Região de Chapecó- Unochapecó. \\ Itapiranga, Santa Catarina- Brasil. \\ cplink@unochapeco.edu.br \\ Givanildo Silva \\ Doutor em Ciências Contábeis e Administração, \\ Universidade Regional de Blumenau- FURB \\ Chapecó, Santa Catarina- Brasil. \\ givanildo.silva@unochapeco.edu.br \\ Rodrigo Barichello \\ Doutor em Engenharia de Produção \\ Universidade Federal de Santa Catarina-UFSC \\ Chapecó, Santa Catarina- Brasil. \\ rodrigo.b@unochapeco.edu.br
}

\section{Resumo}

Os projetos públicos devem atentar aos fatores críticos para obterem sucesso mediante os impactos sociais, econômicos e ambientais. Portanto, este artigo tem o objetivo de analisar a interdependência entre os fatores críticos de sucesso no gerenciamento de projetos público sustentáveis. As bases teóricas tratam do gerenciamento de projetos e dos fatores de sucesso dos projetos associados a indicadores sustentáveis. Os dados foram coletados com questionário enviado aos secretários municipais de projetos e planejamento. A amostra da pesquisa compreendeu 100 prefeituras municipais localizadas no estado de Santa Catarina. O tratamento dos dados foi realizado com uso da estatística de correlação de Pearson. Os resultados sugerem que a inovação exerce maior importância nos fatores críticos de sucesso no gerenciamento de projetos sustentáveis. A liderança e a gestão dos recursos também se mostraram relevantes na elaboração de projetos sustentáveis para captação de recursos. Em geral, conclui-se que o sucesso no gerenciamento de projetos sustentáveis está atrelado a visão conjunta dos onze fatores críticos de sucesso que se relacionaram entre si, com destaque para os objetivos do projeto e o controle dos processos. Os resultados sugerem que o sucesso no gerenciamento de projetos sustentáveis depende da atenção dada em todos fatores críticos, comprovando empiricamente a teoria prevista na literatura.

Palavras-chaves: Projetos Sustentáveis. Gestão de Projetos. Projetos Públicos. Sustentabilidade.

\begin{abstract}
Public projects must pay attention to the critical factors to be successful through social, economic and environmental impacts. Therefore, this article aims to analyze the interdependence between the critical success factors in the management of sustainable public projects. The theoretical bases deal with project management and project success factors associated with sustainable indicators. The data were collected with a questionnaire sent to the municipal secretaries of projects and planning. The research sample comprised 100 city halls located in the state of Santa Catarina. Data processing was performed using Pearson's correlation statistics. The results suggest that innovation plays a greater role in the critical success factors in managing sustainable projects. Leadership and resource management were also relevant in the development of sustainable projects to raise funds. In general, it is concluded that
\end{abstract}


the success in the management of sustainable projects is linked to the joint vision of the eleven critical success factors that were related to each other, with emphasis on the objectives of the project and the control of the processes. The results suggest that success in the management of sustainable projects depends on the attention given to all critical factors, empirically proving the theory foreseen in the literature.

Keywords: Sustainable Projects. Project Management. Public Projects. Sustainability.

\section{Cite como - American Psychological Association (APA)}

Link, C. P., Silva, G., Barichello, R., \& Dal Magro, C. B. (2020, maio/ago.). Fatores críticos no gerenciamento de projetos públicos sustentáveis. Revista de Gestao e Projetos (GeP), 11(2), 87-109. https://doi.org/10.5585/gep.v11i2.17490.

\section{Introdução}

O Project Management Institute (2013) define que o projeto se estabelece pelo esforço temporário para criar um produto, serviço ou resultado exclusivo. Os projetos têm assumido papel central no desenvolvimento de mudanças e alternativas aos negócios, deixando a premissa de utilidade exclusiva à resolução de problemas técnicos (Andersen \& Jessen, 2003).

Para auxiliar neste processo, existem ferramentas de gerenciamento dos projetos que desenvolvem habilidades para planejar, implementar e gerenciar todas as etapas de um projeto (Andersen \& Jessen, 2003). A eficiência no gerenciamento de projetos pode garantir o sucesso de um projeto (Jha \& Iyer, 2006).

Os projetos para o setor público são tão importantes quanto aqueles executados pelas empresas privadas, principalmente pela forte associação entre os projetos públicos com a sociedade que será beneficiada com sua implementação (Rabechini Jr. \& Carvalho, 2006; Silva, Teixeira \& Lima Rodrigues, 2016). Por meio dos projetos públicos é possível o Estado encaminhar recursos para empreender grandes necessidades da gestão pública, tendo como principal intuito o atendimento das necessidades e interesses da comunidade e do grande coletivo da sociedade (Pestana \& Valente, 2010).

Atualmente, o gerenciamento de projetos é utilizado por organizações dos mais diferentes ramos de atividade, até mesmo na área pública, e tem sido de suma importância para reverter o planejamento em resultados, conseguindo otimizar a alocação de recursos, para diminuir as surpresas, fazendo com que o gerenciamento de projetos seja mais efetivo e relevante (Pestana \& Valente, 2010).

Portanto, para que a gestão pública consiga realizar uma gestão dos recursos adquiridos por meio da realização de projetos públicos de uma maneira eficiente torna-se 
necessário o gerenciamento de projetos. Nesse sentido, a eficiência na gestão de recursos é fundamental para cumprir as metas delineadas no escopo do projeto (Rabechini Jr. \& Carvalho, 2006; Damasceno Jr. \& Chaves, 2017). A utilização de boas práticas em gerenciamento de projetos no setor público é ainda muito recente. Contudo, a cobrança crescente dos cidadãos por serviços públicos de qualidade robustece a importância de práticas eficientes em todas as esferas do poder público (Pestana \& Valente, 2010).

Verifica-se que tais implicações reforçam a relevância de se investigar os projetos públicos, tendo como finalidade analisar os fatores considerados críticos do gerenciamento de projetos públicos buscando com que os mesmos sejam sustentáveis. Levando em consideração que a sustentabilidade está cada vez mais presente no mundo corporativo (Barbieri, Vasconcelos, Andreassi \& Vasconcelos, 2010). Contudo, tem sido um desafio alinhar o tema da sustentabilidade aplicado à área de projetos (Martens, Brones \& Carvalho, 2013). Nesse sentido, as atividades de gerenciamento de projetos também precisaram se adaptar ao ambiente de sustentabilidade nas dimensões econômica, ambiental e social (Carvalho \& Rabechini Jr., 2011; Martens \& Carvalho, 2017).

Atualmente as organizações buscam por procedimentos e ferramentas que apoiem a gestão de forma sustentável e que os três níveis organizacionais - estratégico, tático e operacional - devem implementar o tripé da sustentabilidade levando em consideração aspectos sociais (pessoas), ambientais (planeta) e econômicos (lucro ou resultados) (Fernández-Sánchez \& Rodríguez-López, 2010; Sabino \& Rabechini Jr., 2012). Os autores ainda explicam que é no nível tático que está a gerência de projetos e neste nível é onde menos se encontram evidências da aplicação dos conceitos de sustentabilidade.

A execução de projetos busca melhorar a eficiência e a efetividade no uso dos recursos da organização, sejam eles humanos ou financeiros, e com o trabalho fluindo horizontalmente, por meio de processos ou fluxos de trabalho. Como a preocupação da sociedade está na sustentabilidade, busca-se então unir projetos que sejam sustentáveis por si só e que seus resultados permitam o desenvolvimento organizacional sustentável, atendendo ao tripé da sustentabilidade: econômico, social e ambiental (Sabino \& Rabechini Jr., 2012, Martens, Brones \& Carvalho, 2013).

Diante das exposições, surge a seguinte questão problema: Quais são os fatores críticos do gerenciamento de projetos sustentáveis no setor público? Em busca de uma resposta para o questionamento, o estudo tem o objetivo de analisar a interdependência entre os fatores críticos de sucesso no gerenciamento de projetos público sustentáveis.

Tendo como justificativa desse estudo a constante presença da sustentabilidade no 
contexto social atual, além de ser uma constante preocupação das empresas públicas e privadas, a competição saudável no mercado, nota-se que muitas práticas de sustentabilidade resultam em forças que motivam o setor público à empreender de acordo com as necessidades e interesses que a comunidade possui, tendo como objetivo principal, o atendimento das necessidades da sociedade no coletivo (Pestana \& Valente, 2010; Oliveira, Queiroz \& Moreira, 2014). Sabino e Rabechini Jr. (2012), Brones e Carvalho (2015), entre outros, ressaltam que mesmo que a sustentabilidade esteja sendo discutida desde a década de 1970, é possível observar que ainda há poucas pesquisas que busquem realizar um alinhamento entre a gerência de projetos e a sustentabilidade.

Reiterando a relevância do estudo, é levado em consideração o triple bottom line da sustentabilidade, que destaca as áreas ambiental, econômica e social (Elkington, 2012), sendo considerada uma necessidade de que as pesquisas que abordem o tripé da sustentabilidade enfoquem nas relações entre a sustentabilidade e a gestão de projetos (Brones \& Carvalho, 2015; Marcelino-Sádaba, González-Jaen \& Pérez-Ezcurdia, 2015; Cluzel, Yannou, Millet \& Leroy, 2016; Luiz, Jugend, Jabbour, Luiz \& Souza, 2016). A prática de gestão de projetos está passando por adaptações no sentido de oferecer maior sustentabilidade (Abidin \& Pasquire, 2007; Martens \& Carvalho, 2017).

\section{Fundamentos teóricos}

A partir da revisão de literatura, torna-se possível realizar um aprofundamento do estudo e fornecer maior consistência sobre o gerenciamento de projetos, os projetos públicos, os fatores críticos de sucesso do projeto e os indicadores sustentáveis.

\subsection{Gerenciamento de projetos e os projetos públicos}

Com os avanços tecnológicos, torna-se cada vez mais desafiador o fato de a alta administração desenvolver e aprimorar a capacidade de ampliar um portfólio de projetos que se enquadre no modelo de organização da empresa, para que de modo eles possam contribuir com o alcance dos resultados e benefícios propostos pela empresa (Siqueira, Crispim \& Gaspar, 2017). Contudo, estudos baseados em empresas brasileiras mostraram que poucas têm formalizado e desenvolvido um modelo de gerenciamento do processo de inovação e de projetos (Rabechini Jr., Carvalho \& Laurindo, 2002; Borges \& Carvalho, 2015).

Para Carvalho, Reis \& Cavalcante (2011), a utilização de definições tem ajudado os pesquisadores e interessados no assunto, bem como as organizações a entender a natureza dos projetos. Por serem munidas de um conjunto de conceitos, elas podem revelar características, semelhanças e diferenças dos projetos, assim como suas particularidades. 
Prado (2008) afirma que, o sucesso em gerenciamento de projeto está relacionado ao nível de maturidade das empresas. Ainda relaciona que, a causa para as falhas em projetos não está ligada à falta de recursos financeiros ou tecnológicos, mas à falta de conhecimento em gestão de projeto, maturidade organizacional, fatores contingenciais e características do ciclo de vida adotado, entre outras. Prado (2008) menciona que, a probabilidade de sucesso é de $42 \%$ para um nível de maturidade baixo, de $56 \%$ para maturidade média e de $73 \%$ para maturidade alta. As definições de sucesso são definidas pela organização e devem ser definidas antes de iniciar o projeto, com métricas específicas para verificar se foi alcançado (Almeida \& Farias Filho, 2010).

A definição mais utilizada para fracasso de um projeto é quando este não atinge as metas de desempenho técnico, custo, prazo ou escopo. Em contrapartida um projeto de sucesso é aquele cujo produto é entregue de acordo com o cronograma, os custos estimados e com alto nível de qualidade. A qualidade é vista como atendimento às funcionalidades $\mathrm{e}$ ao desempenho técnico do produto do projeto. Os autores relacionam fracasso à perda do controle das variáveis gerenciais do projeto e por consequência, seu sucesso é fruto do controle efetivo destas variáveis (Cleland \& Ireland, 2002).

O sucesso ou fracasso de um projeto pode ser percebido diferentemente pelas partes interessadas no projeto (stakeholders). Isto expõe a avaliação do desempenho do projeto à subjetividade do olhar de quem o avalia, entretanto, existem fatores que evidenciam o sucesso de um projeto: (1) os objetivos do projeto são cumpridos dentro do prazo e do orçamento; (2) os stakeholders consideram adequados os resultados do projeto e a forma como foram administrados; (3) os membros da equipe consideram a participação no projeto uma experiência valiosa e; (4) o resultado do projeto gerou lucro ou alguma vantagem competitiva para a organização (Cleland \& Ireland, 2002).

Nos setores públicos os projetos apresentam a mesma importância que os projetos executados nos setores privados, principalmente pela forte associação entre os projetos públicos com a sociedade que será beneficiada com sua implementação (Rabechini Jr. \& Carvalho, 2006; Damasceno Jr. \& Chaves, 2017). Os projetos públicos tem como finalidade o encaminhamento de recursos por parte do Estado, objetivando o atendimento de necessidades e interesses da comunidade e do grande coletivo da sociedade (Pestana \& Valente, 2010).

Portanto, para que a gestão pública consiga realizar uma gestão dos recursos adquiridos por meio da realização de projetos públicos de uma maneira eficiente torna-se necessário o gerenciamento de projetos. Nesse sentido, a eficiência na gestão de recursos é fundamental para cumprir as metas delineadas no escopo do projeto (Rabechini Jr. \& Carvalho, 2006; Damasceno Jr. \& Chaves, 
2017). A utilização de boas práticas em gerenciamento de projetos no setor público é ainda muito recente. Contudo, a cobrança crescente dos cidadãos por serviços públicos de qualidade robustecem a importância de práticas eficientes em todas as esferas do poder público (Pestana \& Valente, 2010).

Com a finalidade de atender as necessidades da sociedade, a administração pública tem aprimorado continuamente a qualidade dos serviços. A Gestão de Projetos apresenta-se como uma opção essencial para proporcionar esse ganho de qualidade e efetividade nos setores públicos. A definição de projeto remete à ideia de planejamento e execução de tarefas de forma estruturada, por profissionais qualificados, com objetivo claro e definido, cronograma e orçamento conhecidos, além do prévio estabelecimento de controles e indicadores para avaliação dos resultados alcançados, comparados em função dos previstos. Essas prerrogativas fazem do gerenciamento de projetos um instrumento a serviço da governança, que prima entre outros princípios pela efetividade, economicidade, eficiência, transparência e prestação de contas (Pisa \& Oliveira, 2013).

Viana (2012) destaca que um fator determinante para a gestão de projetos é o monitoramento e controle que, quando bem executados, permitem a aferição, correção e realinhamento das atividades em curso. $\mathrm{O}$ autor também destaca que, no gerenciamento de projetos, há uma grande diferença no tratamento das informações entre as áreas privada e pública. Fatores que influenciam neste sentido são: aspectos legais, remuneração, qualificação da mão de obra, estrutura hierárquica, recursos disponíveis, entre outros.

Assim, no setor público deve haver um profissional que saiba trabalhar com as necessidades dos projetos, o gerente de projetos é o indivíduo designado pela organização para conduzir a equipe responsável por determinado projeto ao alcance dos seus objetivos. Ele deve possuir um conjunto equilibrado de habilidades éticas, interpessoais e conceituais. (PMI, 2013). Segundo Kerzner (2006), o gerente de projetos deve conhecer o universo do negócio no qual está envolvido, a fim de promover a integração do trabalho em toda a organização.

$\mathrm{O}$ gerenciamento de projetos de maneira eficiente acarreta em diversos benefícios para as organizações tanto públicas quanto privadas, contudo é fundamental que as organizações apresentem preocupações com o desenvolvimento sustentável evidenciando assim, o sucesso do gerenciamento de projetos.

\subsection{Fatores de sucesso do projeto e indicadores sustentáveis}

O termo sustentabilidade nas organizações é formado por certa complexidade, pois o mesmo representa um conjunto de atributos sociais, econômicos e ambientais, que devem ser interligados para que a organização possa ser caracterizada como sustentável. Esses atributos apresentam a possibilidade de envolver desde a proteção ao 
meio ambiente até uma maior consciência dos envolvidos com o processo produtivo da organização (Cunha, Spers \& Zylbersztajn, 2011).

Nas últimas décadas notou-se diversas mudanças em relação a busca por vantagens competitivas, o que resultou na concretização do gerenciamento de projetos e concomitantemente uma elevada importância em desenvolver competências, gerando um crescimento na formação de profissionais especializados em gestão de projetos (Carvalho \& Rabechini Jr., 2011; Botke, Jansen, Khapova \& Tims, 2018). Compreende-se que a organização por projeto e os próprios projetos ostentam um papel fundamental para $o$ desenvolvimento e implantação de tecnologias e práticas mais sustentáveis (Silvius \& Schipper, 2014).

É possível verificar a relação da gestão de projetos e os possíveis impactos ambientais causados ao longo do desenvolvimento do ciclo de vida de um projeto. Apresentando assim, uma grande relevância a escolha do portfólio de projetos, buscando continuamente levar em consideração os aspectos ambientais envolvidos durante o processo (Brones, Carvalho \& Zancul, 2014; Silvius \& Schipper, 2014; Sánchez, 2015).

O desenvolvimento de um processo cuidadoso pode fornecer um conjunto de indicadores que constituem um modelo do sistema de interesse, a fim de orientar a seleção, implementação e monitoramento da estratégia (Moldan, Billharz \& Matravers,
1997). O estudo sobre os fatores críticos de sucesso do projeto tornou-se um aspecto importante do gerenciamento de projetos desde a década de 1960. Devido à necessidade da identificação dos fatores de sucesso poder ajudar os gerentes de projeto a medir o desempenho e os resultados tendo a finalidade de alocar corretamente os recursos do projeto (Chua, Kog \& Loh, 1999; Cox, Issa \& Ahrens, 2003).

Há um grande número de pesquisas sobre os fatores de sucesso para gerenciamento de projetos em vários setores da indústria. Daniel (1961) encontrou uma relação entre os fatores de "sucesso" e a "crise da informação gerencial”. Belout e Gauvreau (2004) estudaram os fatores de gestão de recursos humanos no sucesso dos projetos. Fortune e White (2006) usaram o modelo formal do sistema como uma ferramenta de enquadramento para estudar o projeto, que foi dito ter um desempenho melhor do que a abordagem da lista de verificação (checklist). Fortune e White (2006) revisaram 63 publicações e concluíram 27 fatores de sucesso na literatura.

Carvalho e Fadul (2012) em seu estudo analisaram a gestão das Organizações Não Governamentais (ONGs) com a finalidade de identificar os fatores críticos de sucesso que impactam no desenvolvimento, na captação de recursos e na relação com as fontes financiadoras. $\mathrm{O}$ estudo foi aplicado em quatro ONGs na Região Metropolitana de Salvador e posteriormente foi realizada a comparação 
entre elas. Como resultados do estudo, evidenciou-se a existência de uma relação direta entre o ambiente, a estrutura administrativa, o posicionamento estratégico e o nível de qualificação técnica dos profissionais da equipe responsáveis pelos resultados nas áreas de captação de recursos e desenvolvimento de projetos sociais.

Mais recentemente, há um número crescente de pesquisas sobre indicadores sustentáveis para projetos. Jasch e Lavicka (2006) analisaram os efeitos financeiros dos indicadores de desempenho de sustentabilidade nos projetos piloto e descobriram que dois direcionadores de custos são o custo de aquisição de produtos e os dias de trabalho perdidos. Fernández-Sánchez e RodríguezLópez, (2010) forneceram um método para identificar indicadores de sustentabilidade para projetos de infraestrutura na Espanha. Eles aplicaram padrões de gestão de risco para gerar 30 indicadores sustentáveis nas três dimensões: ambiental, social e econômica.

Considerando os aspectos ambientais e sociais, Puig, Wooldridge \& Darbra (2014) identificaram os indicadores de desempenho ambiental para o desenvolvimento portuário sustentável por meio de pesquisas de revisão de literatura e entrevistas. Eles agruparam os indicadores em três categorias, que foram: desempenho de gerenciamento, desempenho operacional e condição ambiental. Wang, Yao, Wu \& Jiang (2015), em seu estudo, verificaram que o gerenciamento sustentável de projetos é realizado a partir do ambiente interno, que envolve os aspectos como o relacionamento em equipe de projetos, recursos humanos, resultado, comunicação, stakeholders, e os aspectos externos que abrangem os recursos, os resíduos, a energia e, a poluição.

Em um estudo realizado por Jugend e Figueiredo (2017), em uma empresa do setor de energia, foi possível verificar que todas as etapas dos projetos são consideradas para a integração da sustentabilidade ambiental no ciclo de vida de seus projetos estando previstos e sendo desenvolvidos procedimentos adequados, buscando assim, permitir a seleção e aprovação dos projetos que são desenvolvidos, além de condicionamentos políticos, busca realizar estudos de impacto ambiental.

A sustentabilidade é difícil de definir devido à complexidade dos sistemas naturais humanos interligados (Beratan, Kabala, Loveless, Martin, \& Spyke, 2004). Alguns definiram o gerenciamento sustentável de projetos a partir do ambiente interno os aspectos incluem relacionamento, equipe de projeto, recursos humanos, resultado, comunicação, gerenciamento de partes interessadas, enquanto outros são baseados em aspectos externos, como recursos, desperdício, energia e, poluição. Pesquisas anteriores sobre gerenciamento de projetos concentram-se principalmente na sustentabilidade do projeto no ambiente externo. Faltam pesquisas sobre os indicadores de avaliação para a sustentabilidade interna do gerenciamento de 
projetos, considerando os impactos de longo prazo do projeto sobre o desenvolvimento sustentável desenvolvimento da equipe do projeto e da organização (Wang, Yao, Wu \& Jiang, 2015).

A gerência de projetos e sustentabilidade está cada vez mais sendo utilizados pelas organizações para obter vantagens competitivas e o alinhamento deles com a estratégia organizacional são fundamentais na atualidade. Todas as organizações precisam executar projetos para atingir seus objetivos estratégicos e os autores explicam que tais projetos precisam buscar uma cadeia produtiva sustentável (Rocha, Gomes, Kneipp \& Camargo, 2013). Diante da necessidade de responder as exigências legais, da sociedade e das partes interessadas, as organizações têm buscado incorporar a sustentabilidade em seus planos e projetos e evidenciar em seus relatórios as questões inerentes à sustentabilidade (Rocha, Gomes, Kneipp \& Camargo, 2013).

De modo recente, o gerenciamento de projetos ganhou popularidade nos negócios de serviços, pesquisa institutos, organizações sem fins lucrativos e setores públicos. A partir desse desenvolvimento e almejando uma sociedade em que a sustentabilidade esteja presente nas três dimensões, é necessário que os órgãos públicos realizem o gerenciamento de projetos, visando o desenvolvimento sustentável.

Em seguida, são apresentados os procedimentos metodológicos, com as técnicas e os processos, que traçam o caminho para a realização da pesquisa.

\section{Procedimentos metodológicos}

Para atender ao objetivo do estudo, utilizou-se uma pesquisa caracterizada como descritiva. Sampieri, Collado e Lucio (2013) afirmam que o estudo descritivo busca especificar as propriedades, as características e os perfis de pessoas, de comunidades, de grupos, de processos, de objetos ou de quaisquer outros fenômenos que possam ser submetidos a uma análise.

O método utilizado no desenvolvimento da pesquisa foi a pesquisa de levantamento ou survey. Possibilitando assim, uma forma de alcance de dados ou informações sobre as características ou as opiniões de determinado grupo de pessoas, considerado como representante de uma população-alvo, utilizando um questionário como instrumento de pesquisa, nesse tipo de pesquisa, o respondente não é identificável, portanto o sigilo é garantido (Fonseca, 2002).

Quanto à abordagem do problema, o estudo é quantitativo, pela interpretação dos dados numéricos coletados, que podem ser tratados pela aplicação de métodos e técnicas estatísticas (Theófilo \& Martins, 2016).

Como população da pesquisa teve-se o total de 295 municípios pertencentes ao Estado de Santa Catarina, sendo a amostra do estudo delineada em um total de 100 municípios que retornaram com $\mathrm{o}$ questionário válido, representando assim um total de 33,9\% do 
conjunto de elementos da população. Optou-se por analisar essa população como forma de avaliar o processo de gestão de projetos em instituições públicas, levando em consideração, a utilização de todos os fatores possibilitando assim, analisar se as mesmas possuem preocupação em relação ao gerenciamento eficiente e considerando aspectos sustentáveis. O período de coletas de dados foi de outubro de 2017 a fevereiro de 2018.

Os questionários foram encaminhados aos Secretários de Projetos e Planejamento das prefeituras dos municípios do Estado de Santa Catarina. O primeiro contato foi feito por telefone e, posteriormente, o questionário foi enviado ao endereço eletrônico de cada participante da pesquisa em formulário elaborado no Google Forms. Também foi encaminhado o termo de consentimento livre e esclarecido, que autoriza os autores a procederem à divulgação das informações coletadas, de forma agregada.

$\mathrm{O}$ instrumento de pesquisa foi um questionário estruturado, elaborado com base no estudo de Wang, Yao, Wu \& Jiang (2015), os quais identificaram onze fatores críticos para o gerenciamento de projetos sustentáveis.
A interpretação de cada fator foi realizada a partir da aplicação de 38 perguntas estruturadas com o propósito de avaliar a importância desses fatores.

Devido o estudo base ter sido aplicado em forma de entrevista estruturada, levou-se em consideração o princípio da replicabilidade, possibilitando assim, a avaliação de uma amostra maior, por meio da utilização de uma survey. Ainda, foi utilizado como base para replicação por conter elementos considerados suficientes sobre fatores que constituem aspectos e práticas consistentes para avaliação da gestão de projetos nas organizações públicas. Ainda, leva-se em consideração o fato do estudo base, ter definido onze fatores críticos do gerenciamento de projetos, que permitem um desenvolvimento pessoal dos membros da equipe no processo de gerenciamento de projetos como também a melhoria corporativa, por meio da análise de oportunidades sustentáveis.

Sendo assim, o Quadro 1 mostra o constructo da pesquisa, onde são abordados os 11 fatores críticos do gerenciamento de projetos públicos, e as devidas variáveis utilizadas como forma de avaliação dos fatores. 
Quadro 1 - Construto da pesquisa

\begin{tabular}{|c|c|}
\hline $\begin{array}{l}\text { Fatores críticos de } \\
\text { sucesso: }\end{array}$ & Variáveis analisadas: \\
\hline \multirow{2}{*}{$\begin{array}{l}\text { Liderança nos } \\
\text { projetos }\end{array}$} & Compreensão das expectativas dos clientes e das partes interessadas \\
\hline & Habilidades de liderança dos gestores \\
\hline \multirow{3}{*}{$\begin{array}{l}\text { Comunicação nos } \\
\text { projetos }\end{array}$} & Comunicação com as partes interessadas externas \\
\hline & $\begin{array}{l}\text { Estabelecimento de sistema de comunicação de cima para baixo e de baixo para } \\
\text { cima }\end{array}$ \\
\hline & Ambiente de comunicação aberto dentro da equipe do projeto \\
\hline \multirow{3}{*}{$\begin{array}{l}\text { Gerenciamento das } \\
\text { partes interessadas }\end{array}$} & Identificação das partes interessadas e suas necessidades \\
\hline & Controle de qualidade sobre fornecedores e vendedores \\
\hline & Cumprimento das expectativas das partes interessadas \\
\hline \multirow[t]{4}{*}{$\begin{array}{l}\text { Gerenciamento de } \\
\text { equipe de projetos }\end{array}$} & $\begin{array}{l}\text { Na seleção de novos membros da equipe de gestão, identificar e classificar suas } \\
\text { habilidades e necessidades }\end{array}$ \\
\hline & Coordenação da equipe \\
\hline & Levantamento das sugestões dos colaboradores e a respectiva implantação \\
\hline & Engajamento e comprometimento das pessoas da equipe de projeto \\
\hline \multirow[t]{4}{*}{$\begin{array}{l}\text { Gerenciamento de } \\
\text { objetivos de projetos }\end{array}$} & $\begin{array}{l}\text { Estabelecimento de objetivos de projetos específicos, aceitável e facilmente } \\
\text { compreendidos }\end{array}$ \\
\hline & $\begin{array}{l}\text { Estabelecimento de objetivos específicos às pessoas envolvidas no projeto a } \\
\text { partir do objetivo geral do projeto }\end{array}$ \\
\hline & Garantir que cada parte interessada compreenda seus objetos específicos \\
\hline & Permitir que as pessoas conheçam os objetivos e o progresso de outros membros \\
\hline \multirow{2}{*}{$\begin{array}{l}\text { Controle dos } \\
\text { processos de projetos }\end{array}$} & Capacidade de gerenciamento de risco \\
\hline & Verificação constante sobre o processo em relação ao contratado \\
\hline
\end{tabular}




\begin{tabular}{|c|c|}
\hline & Gestores verificam constantemente o progresso do projeto \\
\hline \multirow{5}{*}{$\begin{array}{l}\text { Desenvolvimento } \\
\text { sustentável de } \\
\text { projetos }\end{array}$} & Padronização de documentos e codificação \\
\hline & Sistemas de informações completo e confiável sobre as informações de gestão \\
\hline & Disponibilização de informações aos fornecedores e demais partes interessadas \\
\hline & Resumir e compartilhar as melhores práticas de gestão de informações \\
\hline & Padronização de documentos e codificação \\
\hline \multirow{4}{*}{$\begin{array}{l}\text { Avaliação de } \\
\text { projetos }\end{array}$} & Avaliação do orçamento \\
\hline & Avaliação após a conclusão do projeto \\
\hline & Diminuir a burocracia (reduzindo a complexidade do processo de gestão) \\
\hline & Verificação constante, avaliação e testes referente o progresso do projeto \\
\hline \multirow{4}{*}{$\begin{array}{l}\text { Gestão de recursos } \\
\text { de projetos }\end{array}$} & Uso efetivo do investimento público \\
\hline & Política de economia de energia e recursos \\
\hline & $\begin{array}{l}\text { Compreensão de antecedentes sociais, legais, ambientais e informações técnicas } \\
\text { do projeto }\end{array}$ \\
\hline & Gestão de recursos financeiros \\
\hline \multirow[t]{3}{*}{ Resultado do projeto } & Produtividade do resultado do projeto \\
\hline & Desempenho das perdas e lucros do resultado do projeto \\
\hline & Disposição dos membros da equipe para trabalhar novamente na equipe \\
\hline \multirow[t]{3}{*}{ Inovação } & $\begin{array}{l}\text { Otimizar o gerenciamento de projeto de acordo com as respostas das partes } \\
\text { interessadas }\end{array}$ \\
\hline & Desafios experimentados ou inovação no projeto \\
\hline & Adoção de novas tecnologias e software para gerenciamento de projetos \\
\hline
\end{tabular}

Fonte: Adaptado de Wang, Yao, Wu \& Jiang (2015). 
Para avaliar os fatores críticos relacionados à gestão de projetos públicos sustentáveis, a interpretação de cada fator crítico foi realizada por meio de perguntas estruturadas e foi utilizada escala Likert de 5 pontos, sendo: (1) Sem importância; (2) Pouco importante; (3) Importante; (4) Muito importante; (5) Extremamente importante. Sendo assim, possível avaliar a importância de acordo com cada fator.

Por fim, cabe destacar que a análise dos dados foi elaborada pela formação de um indicador para cada fator crítico. Esse indicador foi construído considerando a média das respostas das questões que compreendem cada fator crítico e, posteriormente, categorizando os fatores por uma classificação dos indicadores médios. Por fim, com a finalidade de investigar a relação entre os fatores de sucesso dos projetos foi elaborada a correlação de Pearson entre os indicadores formados, aplicada com uso do software Statistical Package for the Social Sciences (SPSS), versão 21. O perfil dos respondentes foi analisado através da estatística descritiva e análise univariada dos dados, que compreende análises de média, frequência e percentagem dos resultados encontrados.

\section{Discussão e análise dos resultados}

Esta seção contém a descrição e análise dos dados coletados. Os participantes da pesquisa foram os secretários de planejamento e projetos ou outros profissionais que estivessem ligados com a área de projetos públicos, como engenheiros, diretores, assessores de projetos, arquitetos, prefeitos, chefes, assistentes administrativos, coordenadores técnicos de planejamento estratégico e captação de recursos, estagiários e técnicos em desenvolvimento. A população participante da pesquisa é de 295 prefeituras do Estado de Santa Catarina e, a amostra compreende 100 prefeituras municipais, portanto, $33,90 \%$ que participaram da pesquisa.

Para identificar as características da amostra foram levantadas diferentes informações, tais como: cargo ocupado, tempo de trabalho na atividade de projetos e formação escolar. Verificou-se que a maioria dos respondentes (92\%) são os secretários, engenheiros, diretores, assessores de projetos, arquitetos e prefeitos. Em relação ao tempo de trabalho, a estratificação dos participantes da pesquisa mostrou que $85 \%$ da amostra participante da pesquisa possui mais de 3 anos de atividades em projetos. Considerando a formação escolar, evidenciou-se que $91 \%$ possuem ensino superior completo.

Em relação ao questionário aplicado, foi utilizada uma adaptação do estudo de Wang, Yao, Wu e Jiang (2015), onde foram identificados 11 fatores críticos para $o$ gerenciamento de projetos sustentáveis. Para cada fator, a pontuação média das questões relativas foi tomada como a importância para o Gerenciamento de projetos. Os 11 fatores foram classificados com base em sua pontuação média. A Tabela 1 apresenta a 
pontuação dos fatores relacionados ao sucesso do processo de projetos.

Tabela 1 - Classificação dos Fatores Críticos de Sucesso

\begin{tabular}{ccc}
\hline Classificação & Fator & Importância \\
\hline 1 & Inovação & 4,13 \\
2 & Liderança & 4,12 \\
3 & Gestão de recursos & 4,10 \\
4 & Resultado & 4,04 \\
5 & Partes Interessadas & 4,00 \\
6 & Avaliação & 3,95 \\
7 & Controle & 3,94 \\
8 & Desenvolvimento & 3,94 \\
9 & sustentável & 3,89 \\
10 & Equipe & 3,86 \\
11 & Objetivo & 3,81 \\
\hline
\end{tabular}

Fonte: Dados da pesquisa (2018).

O resultado mostra que a inovação é o fator mais significativo na sustentabilidade da gestão de projetos, que gerou 4,13 pontos. A inovação é importante no desenvolvimento de projetos por ser considerada como elemento diferenciador das organizações que buscam aumentar a competitividade (Carvalho, Reis \& Cavalcante, 2011). Para que a inovação seja incorporada à cultura de uma organização, é necessário encará-la como um processo que pode ser conduzido, com a finalidade de desenvolver e explorar o novo conhecimento, em seus aspectos técnicos e comerciais (Rabechini Jr., Carvalho \& Laurindo, 2002).

O desenvolvimento da liderança foi avaliado como o segundo fator mais importante, essa importância é devido ao líder ser uma pessoa visionária quando o rumo do projeto não é conhecido, ser um colaborador quando o consenso é necessário e ser capaz de motivar a sua equipe (Andrade, 2010; Cleland
\& Ireland, 2002). Quando bem aplicada, a liderança no projeto ajuda a formar equipes eficientes e favorece a motivação e a sinergia necessárias para que se possa atingir com êxito os objetivos do projeto (Suzano, Dunham \& Martins, 2013).

A gestão de recursos é outro fator essencial, devido possibilitar para que os órgãos públicos consigam novos recursos em favor de seus munícipes. O gerenciamento de projetos é utilizado por organizações dos mais diferentes ramos de atividade, até mesmo na área pública, e tem sido de suma importância para reverter o planejamento em resultados, conseguindo otimizar a alocação de recursos, diminuindo as surpresas, e trazendo maior eficiência à gestão de projetos (Pestana \& Valente, 2010).

Os fatores menos importantes são: avaliação de projetos, controle de processos, desenvolvimento sustentável, equipe de 
projetos, objetivos do projeto e a comunicação, abaixo de 4 pontos.

A fim de investigar a relação entre os fatores de sucesso dos projetos, a análise de

Tabela 2 - Correlação entre os 11 fatores críticos correlação de Pearson foi realizada usando o software SPSS. A Tabela 2 apresenta a correlação entre os 11 fatores de sucesso.

\begin{tabular}{|c|c|c|c|c|c|c|c|c|c|c|c|}
\hline & $\mathbf{L}$ & C & P.I. & E.P. & O.P. & C.P. & D.S. & A.P & G.R. & R.P. & G.I. \\
\hline $\mathbf{L}$ & 1 &, $520^{* *}$ &, $448^{* *}$ &, $398^{* *}$ &, $441^{* *}$ &, $420^{* *}$ &, $355^{* *}$ &, $358^{* *}$ &, $411^{* *}$ &, $306^{* *}$ &, $244^{*}$ \\
\hline C &, $520^{* * *}$ & 1 &, $510^{* *}$ &, $543^{* *}$ &, $580^{* *}$ &, $413^{* *}$ &, $342^{* *}$ &, $430^{* *}$ &, $357^{* *}$ &, $389^{* *}$ &, $263^{* * *}$ \\
\hline P.I. &, $448^{* *}$ &, $510^{* *}$ & 1 &, $533^{* *}$ & ,601** &, $558^{* *}$ &, $515^{* *}$ &, $526^{* *}$ &, $499^{* *}$ &, $422^{* *}$ &, $398^{* *}$ \\
\hline E.P. &, $398^{* *}$ &, $543^{* *}$ &, $533^{* *}$ & 1 & ,656 &, $513^{* *}$ &, $542^{* *}$ &, $522^{* *}$ &, $366^{* *}$ &, $412^{* *}$ &, $426^{* *}$ \\
\hline O.P. &, $441^{* *}$ &, $580^{* *}$ & ,601** &, $656^{* *}$ & 1 & , $641^{* * *}$ &, $585^{* *}$ &, $469^{* *}$ &, $547^{* *}$ &, $596^{* *}$ &, $523^{* *}$ \\
\hline C.P. &, $420^{* *}$ &, $413^{* *}$ &, $558^{* *}$ &, $513^{* *}$ &, $641^{* *}$ & 1 &, $656^{* *}$ &, $548^{* *}$ &, $624^{* *}$ &, $532^{* *}$ &, $569^{* *}$ \\
\hline D.S. &, $355^{* *}$ &, $342^{* *}$ &, $515^{* *}$ &, $542^{* *}$ &, $585^{* *}$ &, $656^{* * *}$ & 1 &, $589^{* *}$ &, $595^{* *}$ &, $509^{* *}$ &, $531^{* * *}$ \\
\hline A.P. &, $358^{* *}$ &, $430^{* *}$ &, $526^{* *}$ &, $522^{* *}$ &, $469^{* * *}$ &, $548^{* * *}$ &, $589^{* * *}$ & 1 &, $656^{* *}$ &, $579^{* *}$ &, $508^{* *}$ \\
\hline G.P. &, $411^{* * *}$ &, $357^{* *}$ & ,499** &, $366^{* *}$ &, $547^{* *}$ &, $624^{* *}$ &, $595^{* *}$ &, $656^{* *}$ & 1 &, $546^{* *}$ &, $578^{* *}$ \\
\hline R.P. &, $306^{* *}$ &, $389^{* *}$ &, $422^{* * *}$ &, $412^{* *}$ &, $596^{* *}$ &, $532^{* *}$ &, $509^{* *}$ &, $579^{* *}$ &, $546^{* *}$ & 1 &, $653^{* *}$ \\
\hline G.I. &, $244^{*}$ &, $263^{* *}$ & ,398** &, $426^{* *}$ &, $523^{* *}$ &, $569^{* * *}$ &, $531^{* * *}$ &, $508^{* *}$ &, $578^{* *}$ &, $653^{* *}$ & 1 \\
\hline
\end{tabular}

Nota: $\mathbf{L}=$ Liderança; $\mathbf{C}=$ Comunicação; P.I.= Partes interessadas; E.P.= Equipe de projetos; O.P.= Objetivos dos projetos; C.P.= Controle de projetos; D.S.= Desenvolvimento sustentável; A.P.= Avaliação de projetos; G.P.= Gestão de Projetos; R.P = Resultado do projeto; G.I = Gestão de Inovação.

Fonte: Dados da pesquisa (2018). 
Verifica-se que a liderança apresenta correlação significativa com a comunicação, com as partes interessadas, com os objetivos do projeto, do controle de projetos e com a gestão de recursos. Os achados são confirmados pelo estudo realizado por Russo, Ruiz e Cunha (2005), que um líder precisa ter flexibilidade e diferentes habilidades, para conseguir ter sucesso em características como segmento, tamanho da equipe, tamanho do orçamento e prazo. Em momentos diversos exigem-se habilidades distintas para cada fase do projeto. Contudo, nota-se que a liderança não é significativa em relação a equipe de projetos, ao desenvolvimento sustentável, a avaliação de projetos, os resultados de projetos e a gestão da inovação.

A comunicação também apresenta correlação significativa com a liderança, as partes interessadas, a equipe de projetos, o objetivo do projeto, o controle de projetos e a avaliação do projeto. Nas últimas décadas notou-se um elevado avanço na busca por vantagens competitivas, o que resultou na concretização do gerenciamento de projetos e concomitantemente, uma elevada importância em desenvolver competências como a comunicação, a liderança, entre outras, gerando um crescimento na formação de profissionais especializados em gestão de projetos (Carvalho \& Rabechini Jr, 2011; Botke, Jansen, Khapova \& Tims, 2018). A comunicação tem uma correlação pouco significativa em relação ao desenvolvimento sustentável em projetos, a gestão de recursos, os resultados dos projetos e a gestão da inovação.

Em relação ao fator de partes interessadas, averígua-se que há correlação significativa com os fatores de liderança, comunicação, equipe de projetos, controle de projetos, desenvolvimento sustentável, avaliação de projetos, gestão de recursos e resultados de projetos. É possível notar que há uma correlação mais forte em relação aos objetivos dos projetos. Isso se confirma com Wang, Yao, Wu e Jiang (2015), que verificaram que o gerenciamento sustentável de projetos é realizado a partir do ambiente interno, e envolve os aspectos de relacionamento em equipe de projetos, recursos humanos, resultado, comunicação, stakeholders, e os aspectos externos que abrangem os recursos, os resíduos, a energia e a poluição. Os autores destacam que estudos realizados anteriormente sobre $\mathrm{o}$ gerenciamento de projetos concentraram-se unicamente na sustentabilidade de projetos no ambiente externo. Contudo, é necessário observar que as partes interessadas não se correlacionam com a gestão da inovação.

$\mathrm{O}$ fator relacionado a equipe de projetos apresenta correlação significativa aos fatores de comunicação, partes interessadas, controle de projetos, desenvolvimento sustentável, avaliação de projetos, resultados dos projetos e gestão da inovação, e apresenta uma correlação diferenciada em relação aos objetivos do projeto, de maneira similar como a correlação entre as partes interessadas e os 
objetivos do projeto avaliadas anteriormente. Tal fato é comprovado pelo estudo realizado por Lucchesi (2013), que um gestor de projetos requer integração com a sua equipe, para que possa exercer sua função de organizador do trabalho, pela divisão e acompanhamento de atividades uma a uma, de maneira a maximizar o uso dos recursos humanos. Os fatores que menos se correlacionam com o fator de equipe de projetos é a liderança e a gestão de recursos.

Analisando o fator objetivo do projeto, é possível notar que se correlaciona significativamente com todos os outros dez fatores e em destaque apresenta-se a correlação com a equipe de projetos e o controle de projetos. Tal fator deve-se a necessidade do trabalho em equipe e do controle para conseguir alcançar o objetivo proposto pelos órgãos públicos. Para autores Kessler e Winkelhofer (2011), é necessário que o objetivo do projeto seja atingido e os recursos dos projetos sejam planejados na forma de orçamentos, tempo ou capacidades, para que o sucesso do projeto seja efetivado.

O fator controle de projetos de maneira similar apresenta correlação entre todos os demais fatores, contudo de maneira mais significativa com os objetivos dos projetos, com o desenvolvimento sustentável e com o gerenciamento de recursos. Assim, é evidente a importância que a gestão de portfólios de projetos exerce, sendo que, o papel da gestão é realizar uma análise criteriosa em cada projeto, com o objetivo de concretizar os objetivos organizacionais com as perspectivas da sustentabilidade. Considerando os fatores para haver uma gestão harmoniosa dos projetos do portfólio e a busca por recursos, guiando as organizações sobre quais os projetos devem ser aprovados, como torná-los relevantes para que os mesmos sejam priorizados e principalmente quais os projetos devem ser cancelados (Cooper, Edgett \& Kleinschmidt, 1999; Abrantes \& Figueiredo, 2014, 2015).

O desenvolvimento sustentável apresenta correlação com as partes interessadas, a equipe de projetos, os objetivos de projetos, a avaliação de projetos, a gestão de recursos, com o resultado do projeto e com a gestão da inovação. Encontra-se respaldo em relação a preocupação do desenvolvimento sustentável em um estudo realizado por Jugend e Figueiredo (2017) em empresa do setor de energia, verificando que todas as etapas dos projetos são consideradas para a integração da sustentabilidade ambiental no ciclo de vida de seus projetos estando previstos e sendo desenvolvidos procedimentos adequados, buscando assim, permitir a seleção e aprovação dos projetos que são desenvolvidos, que além de condicionamentos políticos, busca realizar estudos de impacto ambiental. Destaca-se que o desenvolvimento sustentável não se correlaciona com a liderança e com a comunicação.

A avaliação de projetos correlaciona com 9 fatores, entre eles a comunicação, às partes interessadas, a equipe de projetos, os objetivos dos projetos, o controle de projetos, o desenvolvimento sustentável, o resultado do 
projeto e com a gestão da inovação. De maneira mais relevante é a correlação entre a avaliação de projetos e a gestão de recursos. A avaliação de projetos é composta por três critérios, sendo o atendimento do orçamento que busca efetivamente avaliar o valor previsto orçado em relação ao valor final orçado do projeto, o critério de atendimento do prazo busca avaliar a diferença em dias corridos do prazo inicial do projeto em relação ao prazo final do projeto e a variação da margem, é considerada a variação percentual da margem de valor final do projeto em relação ao valor inicialmente planejado (Patah, 2010).

$\mathrm{O}$ fator designado de gestão de recursos apresenta correlação com 9 fatores, como a liderança, partes interessadas, equipe de projetos, objetivos de projetos, desenvolvimento sustentável, gestão de recursos, resultado do projeto. Destaca-se a correlação entre a gestão de recursos com o controle de projetos e com a avaliação de projetos. A partir dos resultados encontrados é possível verificar que é notável a relação da gestão de projetos e os possíveis impactos ambientais causados ao longo do desenvolvimento do ciclo de vida de um projeto. Apresentando assim, uma grande relevância a escolha do portfólio de projetos, buscando continuamente levar em consideração os aspectos ambientais envolvidos durante o processo (Brones, Carvalho \& Zancul, 2014; Silvius \& Schipper, 2014; Sánchez, 2015). Ainda é possível verificar que não há correlação da gestão de recursos com a comunicação.

O resultado do projeto proporciona correlação entre as partes interessadas, a equipe de projetos, os objetivos de projetos, o controle de projetos, o desenvolvimento sustentável, a avaliação de projetos e a gestão de recursos, além de apresentar correlação significativa com a gestão da inovação. Para Carvalho e Rabechini (2006) e Martens, Brones e Carvalho (2013), de maneira geral, todas as empresas vivem de projetos, até aquelas cujo produto final não seja determinado por projetos. As atividades inteligentes de projetos são responsáveis por $25 \%$ do produto interno bruto mundial, o que representa algo em torno de US\$ 10 trilhões. Avalia-se que mundialmente 16,5 milhões de pessoas estejam envolvidos com atividades de projetos. Assim, torna-se evidente que o fator resultado do projeto é bastante importante no desenvolvimento mundial.

Por fim, a gestão da inovação é correlacionada com oito fatores, como partes interessadas, equipe de projetos, objetivos de projetos, controle de projetos, desenvolvimento sustentável, avaliação de projetos e gestão de recursos, ainda é possível verificar que há uma correlação com o resultado do projeto. Esse fato é comprovado pelos estudos realizados por Rabechini Jr., Carvalho e Laurindo (2002) e Schelini, Martens, Piscopo e Garcez (2017), que afirmam que, para uma empresa ter vantagem competitiva em relação a inovação e a 
internacionalização, é fundamental que gerem competências em projetos, sendo que para atingir sucesso em projetos é necessário balancear as expectativas dos interessados aos recursos disponíveis, pela utilização de conceitos, ferramentas e técnicas para obter a excelência na gestão de projetos.

\section{Considerações finais}

O estudo teve como propósito analisar os fatores críticos que podem impactar no gerenciamento de projetos sustentáveis no setor público. A pesquisa teve como população um total de 295 municípios do Estado de Santa Catarina, e a amostra foi delineada por 100 indivíduos que retornaram com os questionários. Os resultados mostram que a inovação é o fator mais significativo na sustentabilidade da gestão de projetos, sendo considerado o fator mais importante na adequação do gerenciamento de projetos. Esse fator torna-se um diferencial competitivo, devido a necessidade que se possui em inovar no setor público, conseguindo assim, novos recursos e de maneira diferenciada.

Em seguida, os fatores de liderança e de gestão de recursos, foram fatores considerados de grande importância para o desenvolvimento de um gerenciamento de projetos eficiente. Enquanto que os fatores menos importantes são a avaliação de projetos, o controle de processos, o desenvolvimento sustentável, a equipe de projetos, os objetivos do projeto e a comunicação, contudo, esses fatores não deveriam ser os menos importantes, pois todos fazem parte de um conjunto que juntos tornam o gerenciamento de projetos públicos mais efetivos.

A análise apresenta que os 11 fatores críticos do gerenciamento de projetos sustentáveis se correlacionam entre si, destacando os objetivos do projeto e o controle de processos que correlacionam com todos os demais fatores, evidenciando que é necessário haver todas as etapas no desenvolvimento de projetos públicos sustentáveis.

Um estudo desta natureza é importante devido proporcionar um diagnóstico do gerenciamento de projetos sustentáveis, sendo possível avaliar os fatores relevantes e realizar uma perspectiva em relação aos possíveis impactos causados pela falta de um gerenciamento de projetos efetivo. Podendo o estudo ser uma ferramenta de utilidade para gestores no momento da tomada de decisões e construção de um plano de ação que possa ser relevante no desenvolvimento de atuais ou futuros projetos.

Em relação a pesquisas futuras sugerese a aplicação em empresas específicas do setor de gerenciamento de projetos buscando investigar os fatores críticos do gerenciamento de projetos em um todo, para assim, poder melhorar o desempenho dos projetos.

\section{Referências}

Abidin, N. Z., \& Pasquire, C. L. (2007). Revolutionize value management: A mode towards sustainability. International Journal of Project Management, 25(3), 275-282. 
Abrantes, R., \& Figueiredo, J. (2014). Feature based process framework to manage scope in dynamic NPD portfolios. International Journal of Project Management, 32(5), 874884.

Abrantes, R., \& Figueiredo, J. (2015). Resource management process framework for dynamic NPD portfolios. International Journal of Project Management, 33(6), 1274-1288.

Almeida, N. O., \& Farias, J. R. (2010). Definindo sucesso em projetos. Revista de Gestão e Projetos-GeP, São Paulo, 1(2), 6885.

Andersen, E. S., \& Jessen, S. A. (2003). Project maturity in organizations. International Journal of Project Management, 21(6), 457-461. http://dx.doi.org/10.1016/S02637863(02)00088-1.

Andrade, E. F. (2010). Gerenciamento de Conflitos em Projetos. Monografia apresentada ao curso MBA em Gerência de Projetos, Universidade Federal Fluminense. Rio de Janeiro, RJ.

Barbieri, J. C., Vasconcelos, I. F. G. D., Andreassi, T., \& Vasconcelos, F. C. D. (2010). Inovação e sustentabilidade: novos modelos e proposições.

Belout, A., \& Gauvreau, C. (2004). Factors influencing project success: the impact of human resource management. International journal of project management, 22(1), 1-11.

Beratan, K.K., Kabala, S.J., Loveless, S.M., Martin, P.J.S., \& Spyke, N.P. (2004), Environmental Monitoring and Assessment 94: 179-191.

Borges, J. G., \& Carvalho, M. M. D. (2015). Critérios de sucesso em projetos: um estudo exploratório considerando a interferência das variáveis tipologia de projetos e stakeholders. Production, 25(1), 232-253.

Botke, J. A., Jansen, P. G., Khapova, S. N., \& Tims, M. (2018). Work factors influencing the transfer stages of soft skills training: A literature review. Educational Research Review, 24, 130-147.

Brones, F., \& de Carvalho, M. M. (2015). From 50 to 1: integrating literature toward a systemic ecodesign model. Journal of Cleaner Production, 96, 44-57.

Brones, F., de Carvalho, M. M., \& Zancul, E. S. de (2014). Ecodesign in project management: a missing link for the integration of sustainability in product development?. Journal of Cleaner Production, 80, 106-118.

Carvalho, A. O., \& Fadul, E. M. C. (2012). Os Fatores críticos de sucesso na gestão de organizações não governamentais. Administração Pública e Gestão Social, 4(2), 148-171.

Carvalho, H. G. D., Reis, D. R. D., \& Cavalcante, M. B. (2011). Gestão da inovação.

Carvalho, M. D., \& Rabechini Jr, R. (2011). Fundamentos em gestão de projetos: construindo competências para gerenciar projetos. São Paulo: Atlas.

Chua, D. K. H., Kog, Y. C., \& Loh, P. K. (1999). Critical success factors for different project objectives. Journal of construction engineering and management, 125(3), 142150.

Cleland, di; Ireland, lr. (2002). Gerência de projetos. Rio de Janeiro: Reichmann \& Affonso Editores.

Cluzel, F., Yannou, B., Millet, D., \& Leroy, Y. (2016). Eco-ideation and eco-selection of $\mathrm{R} \& \mathrm{D}$ projects portfolio in complex systems industries. Journal of Cleaner Production, 112, 4329-4343.

Cooper, R. G., Edgett, S. J., \& Kleinschmidt, E. J. (1999). New product portfolio management: practices and performance. Journal of product innovation management, 16(4), 333-351. 
Cox, R. F., Issa, R. R., \& Ahrens, D. (2003). Management's perception of key performance indicators for construction. Journal of construction engineering and management, 129(2), 142151.

Cunha, C. F. da, Spers, E. E., \& Zylbersztajn, D. (2011). Percepção sobre atributos de sustentabilidade em um varejo supermercadista. RAE-Revista de Administração de Empresas, 51(6), 542552.

Damasceno Jr., J., \& Chaves, M. S. (2017). Explorando o uso do modelo target: a gestão de lições aprendidas na área de projetos em uma instituição governamental de segurança pública. Revista Alcance, 24(1), 147-160.

Daniel, D. R. (1961). Management information crisis. Harvard Business Review, 111-121.

Elkington, J. (2001). Sustentabilidade Canibais Com Garfo e Faca: Triple botton line.

Fernández-Sánchez, G., \& Rodríguez-López, F. (2010). A methodology to identify sustainability indicators in construction project management-Application to infrastructure projects in Spain. Ecological Indicators, 10(6), 1193-1201.

Fonseca, J. J. S. (2002). Metodologia da pesquisa científica. [Apostila] Fortaleza: UEC.

Fortune, J., \& White, D. (2006). Framing of project critical success factors by a systems model. International journal of project management, 24(1), 53-65.

Jha, K. N., \& Iyer, K. C. (2006). Critical determinants of project coordination. International Journal of Project Management, 24(4), 314-322. http://dx.doi.org/10.1016/j. ijproman.2005.11.005.

Jasch, C., \& Lavicka, A. (2006). Pilot project on sustainability management accounting with the Styrian automobile cluster. Journal of Cleaner production, 14(14), 1214-1227.
Jugend, D., \& Figueiredo, J. (2017).

Integrating environmental sustainability and project portfolio management: case study in an energy firm. Gestão \& Produção, (AHEAD), 0-0.

Kerzner, H. (2006). Gestão de projetos: as melhores práticas. 2. ed. Porto Alegre: Bookman.

Kessler, H., \& Winkelhofer, G. (2011). Projektmanagement: Leitfaden zur steuerung und führung von projekten. Springer-Verlag.

Lucchesi, P. Z. (2013). Liderança em gestão de projetos.

Luiz, J. V. R., Jugend, D., Jabbour, C. J. C., Luiz, O. R., \& de Souza, F. B. (2016). Ecodesign field of research throughout the world: mapping the territory by using an evolutionary lens. Scientometrics, 109(1), 241-259.

Marcelino-Sádaba, S., González-Jaen, L. F., \& Pérez-Ezcurdia, A. (2015). Using project management as a way to sustainability. From a comprehensive review to a framework definition. Journal of cleaner production, 99, 1-16.

Martens, M. L., \& Carvalho, M. M. (2017). Key factors of sustainability in project management context: A survey exploring the project managers' perspective. International Journal of Project Management, 35(6), 1084-1102.

Martens, M. L., Brones, F., \& Carvalho, M. M. (2013). Lacunas e tendências na literatura de sustentabilidade no gerenciamento de projetos: uma revisão sistemática mesclando bibliometria e análise de conteúdo. Gestão e Projetos: GeP, 4(1), 165-195.

Moldan, B., Billharz, S., \& Matravers, R. (1997). Sustainability indicators. A report on the project on indicators of sustainable development.

Oliveira C. M., D.; Queiroz S. O., N.; Moreira S., E.. (2014). As práticas de 
sustentabilidade ambiental e suas influências na nova formatação institucional das organizações. Revista de Gestão Ambiental e Sustentabilidade, 3, 3 .

Patah, L. A. (2010). Avaliação da relação do uso de métodos e treinamentos em gerenciamento de projetos no sucesso dos projetos através de uma perspectiva contingencial: uma análise quantitativa (Doctoral dissertation, Universidade de São Paulo).

Pestana, C. V. S., \& Valente, G. V. P. (2010). Gerenciamento de projetos na administração pública: da implantação do escritório de projetos à gestão de portfólio na secretaria de estado de gestão e recursos humanos do Espírito Santo.

Pisa, B.; \& Oliveira, A. (2013). Gestão de projetos na administração pública: um instrumento para o planejamento e desenvolvimento. Seminário Nacional de Planejamento e Desenvolvimento, v. 2,

Prado, D. (2008). Maturidade em gerenciamento de projetos. Nova Lima: INDG Tecnologia e Serviços Ltda, 7.

Project Management Institute - PMI. (2013). Um guia do conjunto de conhecimentos em gerenciamento de projetos: Guia PMBOK$5^{\mathrm{a}}$ Edição.

Puig, M., Wooldridge, C., \& Darbra, R. M. (2014). Identification and selection of environmental performance indicators for sustainable port development. Marine pollution bulletin, 81(1), 124-130.

Rabechini Jr, R., \& Carvalho, M. (2006). Construindo competências para gerenciar projetos. São Paulo: Atlas.

Rabechini Jr, R., Carvalho, M. M., \& Laurindo, F. J. B. (2002). Fatores críticos para implementação de gerenciamento por projetos: o caso de uma organização de pesquisa. Revista Produção, 12(2), 28-41.

Rocha, . C.da. Gomes, C. M. K., Kneipp M.. Camargo, C. R.. (2013) Gestão de projetos e sustentabilidade: um estudo bibliométrico da produção científica na base Web of Science. Revista de Gestão e Projetos - GeP, São Paulo, v. 4, n. 3, p 73-97, set./dez.

Russo, R. D. F. S. M., Ruiz, J. M., \& da Cunha, R. P. (2005). Liderança e influência nas fases da gestão de projetos. Production, 15(3), 362-375.

Sabino, M. De S.. Rabechini Jr., R. (2012). A Contribuição dos Princípios de Sustentabilidade na Gestão de Projetos: $O$ Caso Siemens do Brasil. In: XXVII Simpósio de Gestão da Inovação Tecnológica, v. 1, p. 125. ANPAD.

Sampieri, R. H., Collado, C. F., \& Lucio, P. B. (2013). Metodologia de pesquisa. Penso.

Sánchez, M. A. (2015). Integrating sustainability issues into project management. Journal of Cleaner Production, 96, 319-330.

Schelini, A. L. S., Martens, C. D. P., Piscopo, M. R., \& Garcez, M. P. (2017). A gestão de projetos como vantagem competitiva para internacionalização de empresas brasileiras. Internext, 12(3), 1-15.

Silva, R. R., Teixeira, M. R. S., \& de Lima Rodrigues, F. T. R. (2016). Uma análise da gestão de projetos de extensão de uma Instituição Federal de ensino. Revista de Gestão e Secretariado, 7(3), 150-171.

Silvius, A. J., \& Schipper, R. P. (2014). Sustainability in project management: A literature review and impact analysis. Social Business, 4(1), 63-96.

Siqueira, L. D., Crispim, S. F., \& Gaspar, M. A. (2017). A influência do escritório de projetos e da estrutura organizacional projetizada no alinhamento dos projetos de tecnologia da informação aos modelos de negócios. Revista Gestão \& Tecnologia, 17(2), 66-92.

Suzano, M. A., Dunham, A., \& Martins, H. F.(2013). Contribuição ao Gerenciamento 
do Ciclo de Vida de Projetos: Considerações sobre o Fator Liderança.

Theóphilo, C. R.; Martins, G. A.(2016)

Metodologia da investigação científica para ciências sociais aplicadas. São Paulo: Atlas, 3, 104-119.

Viana, C. P..( 2012) A gestão de projetos no âmbito da Administração Pública Federal: uma visão estratégica. In: Anais do $\mathrm{V}$ Congresso CONSAD de Gestão Pública.

Wang, N.; Yao, S.; N.,;Wu, C. C., \& Jiang, D.. (2015). Critical factors for sustainable project management in public projects. In International Association For Management Of Technology IAMOT 2015 Conference Proceedings. University of Pretoria. 